\title{
Extension of the ELECTRE III Method to the Case of Uncertain Preferences: Application to an Example of the Environmental Management Problem
}

\author{
Mohammed Chaouki ABOUNAIMA ${ }^{1, *}$, Fatima Zahra EL MAZOURI ${ }^{2}$, Loubna LAMRINI ${ }^{1}$, \\ Mohamed OUZARF ${ }^{1}$, Mohammed TALIBI ALAOUI ${ }^{1}$ \\ ${ }^{1}$ Laboratory of Intelligent Systems and Applications, Faculty of Sciences and Technologies, Sidi Mohammed Ben Abdellah University, \\ Fez, Morocco \\ ${ }^{2}$ Université Polytechnique Hauts-de-France, LAMIH UMR CNRS 8201, Valenciennes, France
}

\begin{abstract}
Almost all the decision problems are by nature multi-criteria, where multiple and conflicting criteria must be considered in the decision-making process. For some cases of these decision problems, they are further marked by a lack of certainty and precision in the decision-maker's judgments and preferences. This study aims to propose an extension of the ELECTRE III method, widely used in the multi-criteria decision aid field, to the case of a decision problem where the decision-maker preferences are imprecise and uncertain. In this proposed adaptation, the inaccuracies and uncertainties will be expressed by an uncertainty interval. The proposed extension will be tested with an example problem to demonstrate its feasibility and relevance. In this example of environmental management, we propose to rank three oil refinery installation projects from the best project to the worst project. The experiment results show that the ELECTRE III method extension can be used easily and rigorously when the uncertainty intervals express the decision-maker's preferences. Finally, the results obtained in this work can be extended to other multi-criteria analysis methods.
\end{abstract}

Keywords Multi-Criteria Decision, Aid/Analysis/Making(MCDA/MCDM), Outranking Approach, ELECTRE III method, Uncertainty Interval (UI), Environmental management

AMS 2010 subject classifications 90B50, 68U35

DOI: $10.19139 /$ soic-2310-5070-1194

\section{Introduction}

For more than five decades, multiple multi-criteria decision aid methods have emerged, ranging from the family of weighted sum methods, the best known and used, to the family of outranking methods. Each method family has its advantages and disadvantages. In general, all these methods, called Multi-Criteria Aggregation Procedures (MCAPs)[1], assume that the decision-maker (DM) disposes of certain preferences, so the DM is asked to express the judgments clearly and without any uncertainty and imprecision.

In many real-life situations, these preferences, as well as the judgment values, cannot be provided with precision and certainty. It is empirically demonstrated that decision-maker's judgment often has vague and ambiguous preferences and cannot be estimated with a precise and unique value. Moreover, when we try to make physical measurements of a given natural phenomenon, it is commonly known that there is no physical law that provides exact measurements that all the measures proposed are only approximations.

A physical law will be considered better than another law if it can provide a measure very close to reality. In

\footnotetext{
*Correspondence to: Mohammed Chaouki Abounaima (Email: medchaouki.abounaima@ usmba.ac.ma). Laboratory of Intelligent Systems and Applications, Faculty of Sciences and Technologies, Sidi Mohammed Ben Abdellah University, Fez, Morocco., Road of Imouzzer, 2202, Fez, Morocco.
}

ISSN 2310-5070 (online) ISSN 2311-004X (print)

Copyright (c) 2022 International Academic Press 
all physical law applications, the final result is always expressed using an interval that takes into account the uncertainty in the final measurement. All these remarks about uncertainty and vagueness have been one of the main motivations behind the appearance of fuzzy sets proposed by Lotfi Zadeh[2], the forerunner of fuzzy logic. In this paper, the authors propose an approach that allows the DM to express these judgments using an interval called the uncertainty interval (UI). Indeed, the expression of preferences using a unique value does not reflect the reality of the decision maker's judgments, while the DM can express, without any difficulty, his uncertain and imprecise preferences through these UIs.

This work aims to show how the MCAPs, particularly the ELECTRE (Elimination and Choice Translating the Reality) family methods[3], can be extended and adapted in case the decision maker's preferences are uncertain and imprecise, expressed using UIs. More precisely, the proposed approach consists to extend the method ELECTRE III[4] to rank, from the best to the worst, a set A of actions and alternatives, objects of the problem of decision posed. The approach's principle can be adapted to all other ELECTRE family methods and even the other outranking methods.

In this article, the ELECTRE III method is used because of its ability to allow decision-makers to express their hesitation between the indifference relation I and the preference relation P. For the illustration of the proposed approach, a numerical example, extracted from an environmental management problem, is given at section 5 . In this article, the authors will discuss the uncertain performance in the case of multi-criteria analysis decision problems, and the authors will show how to adapt the ELECTRE III method to the cases of preferences expressed by uncertainty intervals. In the next section, the authors will clarify the problem studied in this paper. In the third section, the authors will present an overview of ELECTRE methods. The fourth section will be devoted to the proposed contribution. Finally, to illustrate the proposed approach, the authors present a practical example of an environmental management problem.

\section{Position of the problem and related works}

The Multi-Criteria Decision Making (MCDM), introduced by B.Roy[5, 6], is a set of methods that help the Decision-Maker (DM) to make decisions using their preferences with multiple and conflictual criteria. Thus, it has been widely applied to various scientific problems, such as the environment [7, 8], industrial [9], land use and management of natural resources [10], mine planning [11], energy management [12], waste management [13, 14], financial and bank management [15], evaluation and selection of projects [16] and transport [17] and many other problems. All the multi-criteria problems require the establishment of a coherent family $\mathrm{F}$ of $\mathrm{m}(\mathrm{m} \geq 2)$ criteria and also require a set $\mathrm{A}$ of $\mathrm{n}(n \geq 1)$ actions or alternatives. Then, the DM must give for all alternative a of $\mathrm{A}$ and for any criterion $g_{j}$ of $\mathrm{F}$, his judgment $g_{j}(a)$. This judgment is also named the preference of the alternative a on the criterion $g_{j}$.

This leads to the establishment of a matrix called preferences matrix or decision matrix $M(n, m)$. This matrix is composed of $\mathrm{n}$ rows and $\mathrm{m}$ columns. The rows represent the alternatives and the columns represent the set of criteria. The majority of MCAPs, especially the ELECTRE family methods proposed by B. Roy [5], require certain preferences $g_{j}(a)$. However, the DMs preferences are usually inherent in imprecision and uncertainty. The preferences are considered uncertain when the DMs have doubts about their validity, and these preferences are considered imprecise when the DMs have difficulty expressing them very clearly and unambiguously.

For example, all the following criteria: "noise pollution", "separation of the territory", "air pollution", "impact on regional planning", used in the practical example, by their physical nature, we cannot express them and measure them with an exact evaluation, whatever the most sophisticated physical material deployed, there will always be uncertainty in this type of measurement.

In order to overcome the lack of MCDM methods that do not accept imprecision, this work proposes an extension of these to allow the use of even imprecise and uncertain preferences. This imprecision and uncertainty will be expressed using an interval called 'uncertainty interval'. To take into account the uncertainty and vagueness that mark certain information, the UIs have been studied and introduced by several mathematical researchers, as in $[18,19]$. 
This work aims to propose an extension of ELECTRE methods to consider uncertain preferences, particularly the authors will focus on the ELECTRE III version. Several related works were realized in the context of uncertainty and imprecision problems. Among these works, we cite the fuzzy MCDM methods such as methods based on fuzzy sets [8], methods based on intuitionistic fuzzy sets [18, 19], and methods based on hesitant fuzzy sets [20, 21]. The majority of these methods are combined with the most popular ELECTRE method to solve a multi-criteria problem with fuzzy information. This list of works is not exhaustive.

As mentioned above, the preferences uncertainty and imprecision are expressed based on UIs. More precisely, for each criterion gj and each action a, the MD can express the evaluation $g_{j}(a)$ by the uncertainty interval: $\left[g_{j}(a)^{-}, g_{j}(a)^{+}\right]$. Where $g_{j}(a)^{-}$and $g_{j}(a)^{+}$are respectively the minimum and maximum limits of the possible variation of the evaluation $g j(a)$. It worth mentioning that $\left[g_{j}(a)^{-} \leq g_{j}(a)^{+}\right]$, and if the DM expresses $g_{j}(a)^{-}=$ $g_{j}(a)^{+}$, then this is a particular case where the evaluation is expressed with a unique and exact value.

\section{Overview of the ELECTRE methods and interval comparison methods}

\subsection{Versions of the ELECTRE methods}

ELECTRE methods are a set of outranking methods based on pairwise comparisons between alternatives. This method is introduced to overcome the disadvantages of "The weighted-sum method". ELECTRE method is not a compensatory method, i.e. a low evaluation on a criterion is not automatic, as the weighted-sum methods, compensated by a high evaluation on another criterion [3]. Moreover, the ELECTRE methods support different measurement scales that can be indifferently cardinal and ordinal [22]. ELECTRE is a family of methods belonging to the outranking synthesis approach. Currently, we find in the literature more than six versions of the ELECTRE methods, each with its characteristics according to whether we adopt true criteria or pseudo-criteria [3], according to the decision problems to be solved and depending on whether one takes into account or not the criteria relative importance, often expressed by weights. The hesitation of the DM between the indifference relation I and the preference relation P B. Roy [1] proposes an extension of the ELECTRE II method, which is the ELECTRE III method, allowing the DM to express the hesitation between indifference and preference among two given alternatives a and $\mathrm{b}$, to do this, two thresholds $p_{j}$ and $q_{j}$ are introduced for each criterion $g_{j}$.

The two thresholds $p_{j}$ and $q_{j}$ may depend on each alternative a, we consider the thresholds $p_{j}\left(g_{j}(a)\right)$ and $q_{j}\left(g_{j}(a)\right)$. B. Roy [1][3] chose a linear form to define these thresholds according to the considered alternative:

$p_{j}\left(g_{j}(a)\right)=\alpha 1 \times g_{j}(a)+\beta 1$ and $q_{j}\left(g_{j}(a)\right)=\alpha 2 \times g_{j}(a)+\beta 2$.

We can opt for any strictly monotonous function to generalize the linear function's choice to calculate the thresholds.

When it is desired to define an indifference area, the first threshold has been introduced, called the indifference threshold, and denoted by the parameter $q$, below which the DM sees no difference between two actions $a$ and $b$ (see Figure 1). Precisely, two actions $a$ and $b$ are considered indifferent, on a given criterion $g_{j}$, when the following inequality (1) is verified:

$$
\left|g_{j}(a)-g_{j}(b)\right|<q_{j}\left(g_{j}(a)\right)
$$

Where $q_{j}$ represents the indifference threshold relative to the $j^{\text {th }}$ criterion $g_{j}$. When it is desired to define an area of hesitation between preference and indifference, a second threshold has been introduced, called the preference threshold, and denoted by the parameter $p_{j}$ with $p_{j} \geq q_{j} \geq 0$. Above this threshold, the DM considers without hesitation that action $a$ is strictly preferred to action $b$ (see Figure 2). Precisely, action $a$ will be considered as preferred without hesitation to the action $b$, on a given criterion $g_{j}$, when the following inequality (2) is verified:

$$
g_{j}(a)-g_{j}(b)>p_{j}\left(g_{j}(a)\right)
$$

$p_{j}$ represents the threshold of preference relative to to the $j^{\text {th }}$ criterion $g_{j}$.

As shown in Figure 2, between the two indifference areas, and preferably, it exists the area of hesitation between 


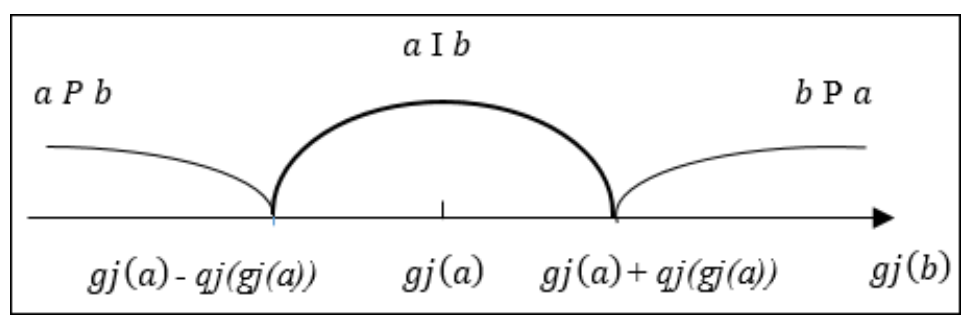

Figure 1. The indifference relation $I$ and the preference relation $P$.

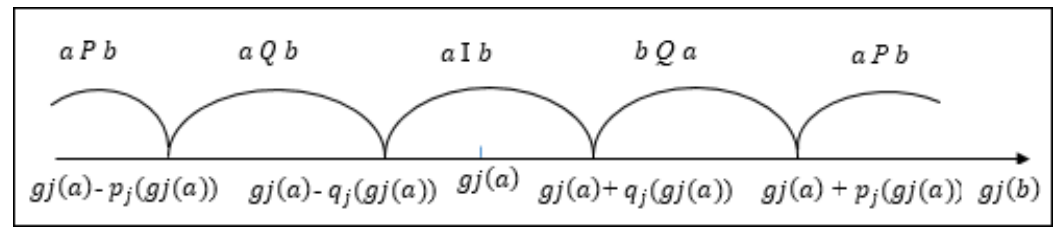

Figure 2. The indifference relation $I$, the weak preference relation $Q$, and the strict preference relation $P$.

preference and indifference $[23,24]$. The weak preference relation $Q$ expresses this area. Then, we can say that action $a$ is weakly preferred to the action $b$, on a given criterion $g_{j}$, when the inequality (3) is satisfied:

$$
q_{j}\left(g_{j}(a)\right)<g_{j}(a)-g_{j}(b)<p_{j}\left(g_{j}(a)\right)
$$

\section{Remarks:}

- When we only consider the indifference threshold (i.e. $0<q_{j}=p_{j}$ ), the considered criterion $g_{j}$ is called "quasi-criterion".

- When the two thresholds are considered (i.e. $0<q_{j}<p_{j}$ ), the considered criterion $g_{j}$ is called "pseudocriterion".

- when the two thresholds are not considered (i.e. $0=q_{j}=p_{j}$ ), the used criterion $g_{j}$ is called "true-criterion".

\subsection{Principle of the ELECTRE III method}

In this study, we use the ELECTRE III method [4] as a method which her objective is to rank potential actions, from the "best" to the "worst" (ranking procedure), although it has a richness and complexity far superior to those of ELECTRE I and ELECTRE II [5], the main approach of this method begins with the Building of the outranking relation $S$ and proceeds to its exploitation by the establishment of two antagonist's pre-orders, the ascending preorder and the descending pre-order. And finally, the two pre-orders are combined and synthesized to obtain a median pre-order as the final result of the method.

Like all ELECTRE procedures, the ELECTRE III version is based on two main concepts: concordance and nondiscordance. The first concept expresses when constructing the outranking relation $S$, the respect of the majority, in the sense that an action $a$ will outrank another action $b$, noted by $a S b$, if the majority of the criteria are in favor, or in concordance, with this assertion $a S b$ [5]. The second concept is eventually to take into account the strong opposition of a minority of criteria, also called the effect of the veto, in the sense that when we search to approve the outranking relation $a S b$ for two given actions $a$ and $b$, if there exists at least one criterion in contradiction with this assertion, the relation $a S b$ will so be rejected.

To fully understand the principle of the method, we give the algorithm of the method in detail in the following paragraph.

\subsection{Algorithm of the basic ELECTRE III method}

Let $A$ be the set of the alternatives $a_{i}(i=1, \ldots, n)$ evaluated according to a set of $m$ points of views $(m \geq 2)$, each point of view $g_{j}(j=1,2, \ldots, m)$ is represented by a set $F$, called criteria. The performance of the alternative $a_{i}$ 
according to the criterion $g_{j}$ is noted by $g_{j}\left(a_{i}\right)$. The different steps of ELECTRE III and calculations are presented below.

- Step 1. Elaboration of the decision matrix:

In any MCDA problem, the first step in the decision process is to provide all alternatives' performance on all criteria. This operation is then summarized in a matrix $M$ called the decision matrix. See equation 4.

- Decision matrix

$$
M=\left(\begin{array}{cccc}
g_{1}\left(a_{1}\right) & g_{2}\left(a_{1}\right) & \ldots & g_{m}\left(a_{1}\right) \\
g_{1}\left(a_{2}\right. & g_{2}\left(a_{2}\right) & \ldots & g_{m}\left(a_{2}\right) \\
\vdots & \vdots & & \vdots \\
g_{1}\left(a_{n}\right) & g_{2}\left(a_{n}\right) & \ldots & g_{m}\left(a_{n}\right)
\end{array}\right)
$$

- Provide the importance vector for each criterion: $W=\left(w_{1}, w_{2}, \ldots, w_{j}, \ldots, w_{m}\right)$. Where $w_{j}$ is the weight of the $j^{t h}$ criterion, which expresses the relative importance of criterion $g_{j}$ compared to the other criteria.

- Provide the indifference $q_{j}$, preference $p_{j}$, and veto $v_{j}$ threshold, where $0<q_{j}<p_{j}<v_{j}$.

- Step 2. The global concordance index $c(a, b)$ is computed for each pair of alternatives $a$ and $b$ by equation 5 .

$$
c(a, b)=\frac{\sum_{j=1}^{m} w_{j} c_{j}(a, b)}{\sum_{j=1}^{m} w_{j}}
$$

Where $c_{j}(a, b)$ is the partial concordance index of the alternative $a$ and the alternative $b$ under the criterion $g_{j}$ expressed by the flowing equation 6 :

$$
c_{j}(a, b)=\left\{\begin{array}{cc}
0 & \text { if } g_{j}(b)-g_{j}(a)>p_{j}\left(g_{j}(a)\right) \\
1 & \text { if } g_{j}(b)-g_{j}(a) \leq q_{j}\left(g_{j}(a)\right) \\
\frac{p_{j}\left(g_{j}(a)\right)-g_{j}(b)}{p_{j}\left(g_{j}(a)\right)-q_{j}\left(g_{j}(a)\right)} & \text { otherwise }
\end{array}\right.
$$

Thus, $0 \leq c_{j}(a, b) \leq 1$.

- Step 3. The discordance index $d_{j}(a, b)$ for each criterion is defined with the flowing equation 7 :

$$
d_{j}(a, b)=\left\{\begin{array}{cc}
0 & \text { if } g_{j}(a)-g_{j}(b) \leq p_{j}\left(g_{j}(a)\right) \\
1 & \text { if } g_{j}(a)-g_{j}(b)>v_{j}\left(g_{j}(a)\right) \\
\frac{g_{j}(a)-g_{j}(b)-p_{j}\left(g_{j}(a)\right)}{v_{j}\left(g_{j}(a)\right)-p_{j}\left(g_{j}(a)\right)} & \text { otherwise }
\end{array}\right.
$$

Thus, $0 \leq d_{j}(a, b) \leq 1$.

- Step 4. Calculation of the credibility degree

The outranking relation is constructed by defining the credibility degree of the assertion $a S b$ as follows:

$$
\rho(a, b)=\left\{\begin{array}{cc}
c(a, b) & \text { if } d_{j}(a, b) \leq c(a, b) \forall g_{j} \in F \\
c(a, b) \times \prod_{g_{j} \in F} \frac{1-d_{j}(a, b)}{1-c(a, b)} & \text { othrewise }
\end{array}\right.
$$

Equation 8 supposes that if the concordance index exceeds that of the discrepancy for all the criteria, the concordance value coincides with that of the credibility degree. Otherwise, we must attenuate the concordance $c(a, b)$ by the obtained discordance. If the discordance index is worth 1 for at least one criterion $g_{j}$, then we have no credibility that $a S b$ is approved and therefore $\rho(a, b)=0$. 
- Step 5. Building and exploitation of the outranking relation $S$

After building the outranking relation $S$ between all alternatives, and to elaborate the ranking of all alternatives, the basic ELECTRE III method uses two intermediate ranking procedures: in the first, the alternatives are ranked from the best to the worst alternative, called "descending distillation", and in the second, the alternatives are ranked from the worst to the best alternative, called "ascending distillation".

The two pre-orders are defined according to a qualification score calculated for each alternative by the following sub-steps [25].

-Sub-step 5.1. In this first sub-step, we define the index $\lambda 0$. This index is equal to the maximum value of all the credibility $\rho(a, b): \rho(a, b)=\max _{a, b \in A} \rho(a, b)$.

-Sub-Step 5.2. In the second sub-step, we calculate now the outranking level $\lambda 1$ which designates the greatest credibility which is lower than the maximum credibility minus a given threshold, called the "discrimination threshold". The outranking level is calculated by the flowing equation 9:

$$
\lambda 1=\max _{\rho(a, b)<\lambda 0-s(\lambda 0) / a, b \in A} \rho(a, b) .
$$

Where $s(\lambda 0)$ expresses the discrimination threshold corresponding to the maximum outranking level $\lambda 0$. The function $s()$ is defined by the formula given by: $s(\lambda)=\alpha \times \lambda+\beta$. Generally, we choose $\alpha=-0.15$ and $\beta=0.3$. At the initial cut, $a$ outrank $b$, if the credibility degree of $a$ and $b$ is strictly greater than the outranking level $\lambda 1$ and exceeds $\rho(a, b)$ increased by the discrimination threshold. This condition is given by:

$$
a S b \Leftrightarrow \rho(a, b)>\lambda 1 \text { and } \rho(a, b)-\rho(b, a)>s(\lambda 1) .
$$

- Sub-Step 5.3. For each relation $a S b$ obtained by the previous step, the alternative $a$ receives a score +1 (strength), however, the alternative $b$ receives a score of -1 (weakness).

For each alternative $a$, the strength is calculated by the function $\varphi^{+}(a)$, and the weakness is calculated by the function $\varphi^{-}(a)$. Then, the two functions are added for calculation the final qualification score noted by $\varphi(a)$. The different scores are obtained by equations 10,11, and 12 as follows [6].

$$
\begin{aligned}
& \varphi^{+}(a)=\sum_{b \in A / a S b}+1 \\
& \varphi^{-}(a)=\sum_{b \in A / b S a}+1 \\
& \varphi(a)=\varphi^{+}(a)-\varphi^{-}(a)
\end{aligned}
$$

- Sub-Step 5.4: Establishment of the pre-order descending: In the descending distillation procedure, the alternative which has obtained the highest qualification score, it is assigned at the first rank, and therefore, it is withdrawn from the procedure, and then the process is repeated for the remaining alternatives. We obtain a first ranking of the alternatives from the best to the worst alternative.

- Sub-Step 5.5: Establishment of the pre-order ascending: In the ascending distillation, the alternative with the lowest qualifying score is assigned first, and therefore, it is removed from the procedure, and then the process is repeated for the remaining alternatives. We obtain a second ranking of the alternatives from the worst to the best alternative. In the end, the ranking obtained is reversed.

- Sub-Step 5.6. Establishment of the final pre-order: The two procedures descending distillation and ascending distillation, are combined to form a final median ranking.

For an illustrative example of the ELECTRE III algorithm, the reader can see our work [6], where we have used the ELECTRE III method for the national electrification program of the Moroccan rural. 


\subsection{Interval Comparison methods}

In the majority of the MCAPs, in particular, the ELECTRE III method, it has been noted that the comparisons between actions for to build the outranking relation are carried out on the real $\mathbb{R}$ line on the basis of the evaluations $g_{j}(a)$ and $g_{j}(b)$. In the proposed approach, we will be called to make the same comparisons, but at the base of the preferences expressed with the UIs: $\left[g_{j}(a)^{-}, g_{j}(a)^{+}\right]$and $\left[g_{j}(b)^{-}, g_{j}(b)^{+}\right]$. To do this, we have looked in the literature, and we have fortunately ended up finding a panoply of work articulated on the comparisons of intervals. As cited before, several works are done in the context of comparing intervals as in [26], I. Alolyan proposed a new method for the comparison of intervals by defining u-ordering, which helps to have an ordering for closed intervals. We can also note the paper [27], which presents a method based on a probabilistic approach for comparing the crisp and fuzzy interval; the article treats all interval location cases by considering the intervals' overlapping. The present work adopts the same idea to compare intervals that represent the preferences of alternatives correspond to each criterion.

\section{The proposed extension of ELECTRE III}

Our extension of the ELECTRE III method is used to solve multi-criteria problems with imprecise preferences of the DM, and whose ultimate goal is to obtain a ranking of the different alternatives. As seen in the algorithm of ELECTRE III method, the man of study, and in consultation with the DM, must begin to construct the matrix of performances $g_{j}(a)$ : for each alternative $a \in A$ and for all criterion $g_{j} \in F$, except in the proposed approach, the performances $g_{j}(a)$ are expressed using a UI: $\left[g_{j}(a)^{-}, g_{j}(a)^{+}\right]$. These intervals will be compared to each other to build the outranking relationship $S$. Moreover, in the basic ELECTRE III method, to take into account the hesitation of the DM between preference and indifference, two thresholds are introduced for each criterion $g_{j}$, named the indifference threshold $q_{j}$ and the preference threshold $p_{j}$. In addition to these two thresholds, we also consider the veto threshold $v_{j}$. All these thresholds are also included in the proposed new approach.

\subsection{Algorithm for the extension of ELECTRE III method}

The proposed algorithm is very similar to that of the basic ELECTRE III method. However, it differs, on the one hand, in the nature of the data of the decision matrix $M$. For our case, we choose UIs as judgement values of the DM. On the other hand, in calculating the partial concordance indices $c_{j}(a, b)$ and calculating the discordance indices $d_{j}(a, b)$, these indices will be calculated according to the intervals' comparisons. All the other steps of the basic ELECTRE III method are similar in the new approach.

\subsection{Definition of the decision matrix}

In a multi-criteria decision problem, it is necessary to define the set $A$ of $n$ alternatives to rank it from the best to the least good. It is the problematic of ranking treated in this work. Also, it is necessary to define a coherent family of $m$ criteria. And as fuzzy and uncertain estimations expressed by intervals of uncertainty, the decision matrix to be constructed must look like the flowing equation 13.

$$
M=\left(\begin{array}{ccc}
{\left[g_{1}\left(a_{1}\right)^{-}, g_{1}\left(a_{1}\right)^{+}\right]} & \ldots & {\left[g_{m}\left(a_{1}\right)^{-}, g_{m}\left(a_{1}\right)^{+}\right]} \\
\vdots & \ddots & \vdots \\
{\left[g_{1}\left(a_{n}\right)^{-}, g_{1}\left(a_{n}\right)^{+}\right]} & \ldots & {\left[g_{m}\left(a_{n}\right)^{-}, g_{m}\left(a_{n}\right)^{+}\right]}
\end{array}\right)
$$

Where $g_{j}\left(a_{i}\right)^{-}$and $g_{j}\left(a_{i}\right)^{+}$are, respectively, the thresholds min and max for the $i^{t h}$ alternative correspond to the $j^{\text {th }}$ criterion. In addition to the decision matrix, as in the ELECTRE III method, the various thresholds and weights for all criteria must also be provided, see Table (1). 
Table 1. Thresholds and weights of criteria

\begin{tabular}{|c|c|c|c|c|c|}
\hline Criteria & $g_{1}$ & $\ldots$ & $g_{j}$ & $\ldots$ & $g_{m}$ \\
\hline Weights of criteria & $w_{1}$ & $\ldots$ & $w_{j}$ & $\ldots$ & $w_{m}$ \\
\hline Indifference threshold & $q_{1}$ & $\ldots$ & $q_{j}$ & $\ldots$ & $q_{m}$ \\
\hline Preference threshold & $p_{1}$ & $\ldots$ & $p_{j}$ & $\ldots$ & $p_{m}$ \\
\hline Veto thresholds & $v_{1}$ & $\ldots$ & $v_{j}$ & $\ldots$ & $v_{m}$ \\
\hline
\end{tabular}

\subsection{Partial concordance calculation}

To calculate the index of concordance $c_{j}(a, b)$ for each criterion $g_{j}$ and each pair of alternatives $(a, b)$, we will be based on a probabilistic method [27]. With the aim to estimate the degree to which the interval $a=\left[g_{j}(a)^{-}, g_{j}(a)^{+}\right]$ will be considered preferred or indifferent to the interval $b=\left[g_{j}(b)^{-}, g_{j}(b)^{+}\right]$, while allowing the possibility of expressing hesitation in the comparison between indifference and the strict preference of the two intervals. In this concordance index calculation, we can distinguish several possible cases according to the first interval's location relative to the second. To compare two alternatives $a$ and $b$, we must distinguish exactly the following six cases.

Case 1: In this first case, we consider that the UI of alternative $b$ is placed before that of alternative $a$. Three possibilities are to envisage, the $\mathrm{UI}=\left[g_{j}(b)^{-}, g_{j}(b)^{+}\right]$is included in the indifference area of $a(a I b)$, included in the weak preference area of $a(a Q b)$, included in the strict preference area of $a(a P b)$, or overlaps with these three areas. In this case, we consider that according to the crière $g_{j}$, alternative $a$ is preferred to the alternative $b$, and we take so $c_{j}(a, b)=1$. Indeed, in this first case, the interval UI of alternative $b$, in the best case, is at least equal to that of alternative $a$. Otherwise, the interval UI of $b$ is included in the area in favor of alternative $a$. See Figure 3. More precisely, if $g_{j}(b)^{+} \leq g_{j}(a)^{+}+q_{j}$ then $c_{j}(a, b)=1$.

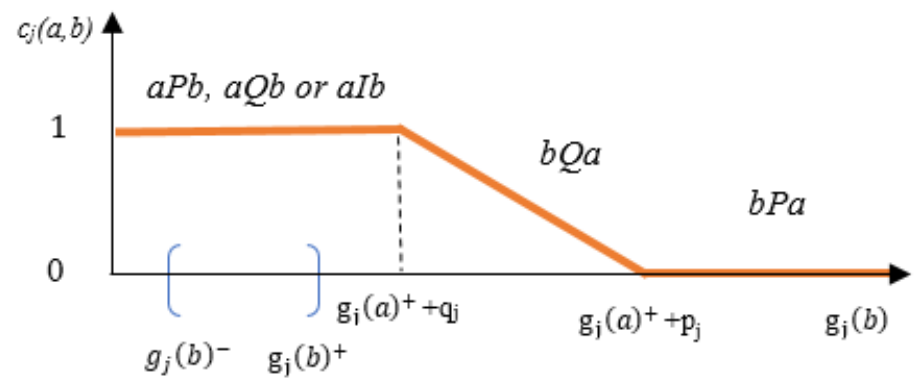

Figure 3. Partial concordance calculation -case 1-

Case 2: In this second case, we consider that the UI of alternative $b$ is located after alternative $a$. We distinguish here the case where the UI of $b$ is included in the preferably weak area of $b(b Q a)$. Therefore, we take as partial concordance the decreasing linear interpolation of the medium $m c$ of the interval $\left[g_{j}(b)^{-}, g_{j}(b)^{+}\right]$, $m c=\frac{\left(g_{j}(b)^{-}+g_{j}(b)^{+}\right)}{2}$, regarding to the weak preference area $\left[g_{j}(a)^{-}+q_{j}, g_{j}(a)^{+}+p_{j}\right]$. See Figure 4 . Precisely: if $g_{j}(a)^{+}+q_{j}<g_{j}(b)^{-}<g_{j}(b)^{+}<g_{j}(a)^{+}+p_{j}$ then $c_{j}(a, b)=\frac{p_{j}+g_{j}(a)^{+}-m c}{p_{j}-q_{j}}$. Property: The proposed formula $c_{j}(a, b)=\frac{p_{j}+g_{j}(a)^{+}-m c}{p_{j}-q_{j}}$ show that the value $c_{j}(a, b)$ varies between 0 and 1 . Moreover, when the UI of alternative $b$ approaches the indifference zone of alternative $a$, the value of $c_{j}(a, b)$ becomes near the maximum concordance 1 . Contrariwise, when the UI of the alternative $b$ approaches the area of preference in favor of $b$, the value $c_{j}(a, b)$ tends towards the minimum value of the concordance 0 . 


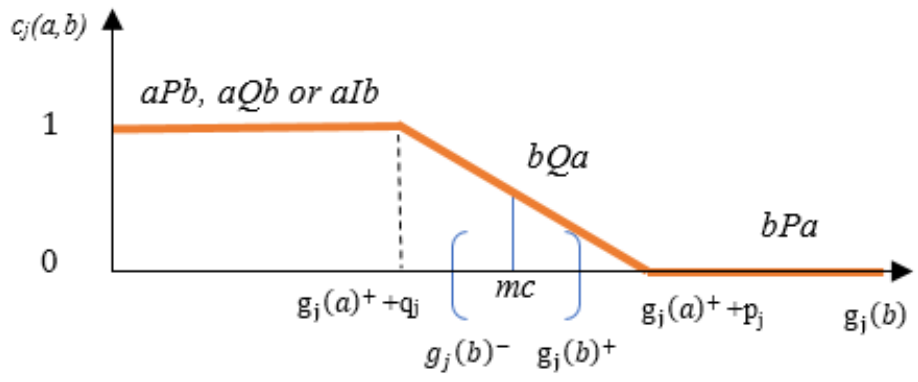

Figure 4. Partial concordance calculation -case 2-

Case 3: In this third case, it is considered that the UI corresponding to alternative $b$ is found after that of alternative $a$. In other words, we distinguish the case where the UI of $b$ is included in the strict preference area of $b(b P a)$. We take in this case $c_{j}(a, b)=0$. See Figure 5.

More precisely: if $g_{j}(b)^{-}>g_{j}(a)^{+}+p_{j}$ then $c_{j}(a, b)=0$.

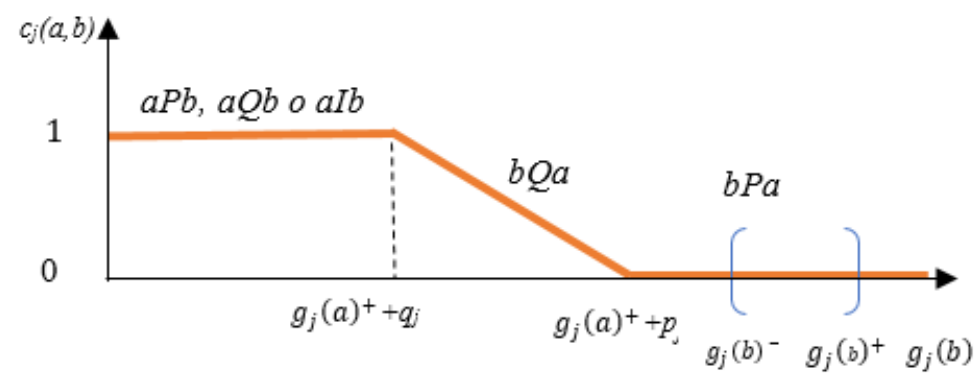

Figure 5. Partial concordance calculation -case 3

Case 4: In this fourth case,we suppose that the UI of alternative $b$ overlaps the area in favor of the alternative $a(a P b, a Q b$ or $a I b)$ and the preference area weak in favor of alternative $b(b Q a)$. In this case, the concordance $c_{j}(a, b)$ must take into account the concordance $c_{j 1}(a, b)$ of the UI of $b$, which is included in the zone in favor of the alternative $a$, and the concordance $c_{j 2}(a, b)$ of the part of the UI of $b$ which is included in the region of preference weak in favor of $b(b Q a)$. See Figure 6.

More precisely: if $g_{j}(b)^{-}<g_{j}(a)^{-}-q_{j}$ and $g_{j}(a)^{+}+q_{j}<g_{j}(b)^{+}<g_{j}(a)^{+}+p_{j}$ then $c_{j}(a, b)=c_{j 1}(a, b)+$ $c_{j 2}(a, b)$.

Where:

- $c_{j 1}(a, b)$ expresses the portion of the concordance of the interval $\left[g_{j}(b)^{-}, g_{j}(a)^{+}+q_{j}\right]$ that is included in the area in favor of $a$. For calculating $c_{j 1}(a, b)$, we apply the case 1, which takes a concordance equal to 1 , but the final result of $c_{j 1}(a, b)$ must be attenuated by the portion of the interval that is not in this area by the value: $1-\frac{g_{j}(b)^{+}-\left(g_{j}(a)^{+}+q_{j}\right)}{g_{j}(b)^{+}-g_{j}(b)^{-}}$.

- $c_{j 2}(a, b)$ expresses the portion of the concordance of the interval $\left[g_{j}(a)^{+}+q_{j}, g_{j}(b)^{+}\right]$, which is included in the weak preference area of $b(b Q a)$. For the calculation of $c_{j 2}(a, b)$, we apply the case 2 , which takes as concordance an interpolation of the medium of the interval $\left[g_{j}(a)^{+}+q_{j}, g_{j}(b)^{+}\right]$. However, the concordance $c_{j 2}(a, b)$ must be attenuated by the region of the UI of $b$ outside the area $b Q a$.

So we take in this case 4 :

$$
c_{j 1}(a, b)=1-\frac{g_{j}(b)^{+}-\left(g_{j}(a)^{+}+q_{j}\right)}{g_{j}(b)^{+}-g_{j}(b)^{-}} \times 1
$$




$$
c_{j 2}(a, b)=\left(1-\frac{\left(g_{j}(a)^{+}+q_{j}\right)-g_{j}(b)^{-}}{g_{j}(b)^{+}-g_{j}(b)^{-}}\right) \times \frac{\left.g_{j}(a)^{+}+p_{j}-m c^{\prime}\right)}{p_{j}-q_{j}}
$$

The equations (14) and (15) are equivalent to :

$$
\begin{gathered}
c_{j 1}(a, b)=\frac{\left(g_{j}(a)^{+}+q_{j}\right)-g_{j}(b)^{-}}{g_{j}(b)^{+}-g_{j}(b)^{-}} \\
c_{j 2}(a, b)=\frac{g_{j}(b)^{+}-\left(g_{j}(a)^{+}+q_{j}\right)}{g_{j}(b)^{+}-g_{j}(b)^{-}} \times \frac{g_{j}(a)^{+}+p_{j}-m c^{\prime}}{p_{j}-q_{j}}
\end{gathered}
$$

Where $m c^{\prime}=\frac{g_{j}(a)^{+}+q_{j}+g_{j}\left(b^{+}\right)}{2}$ is the medium of the interval $\left[g_{j}(a)^{+}+q_{j}, g_{j}\left(b^{+}\right)\right]$. We notice that:

$$
\frac{g_{j}(b)^{+}-\left(g_{j}(a)^{+}+q_{j}\right)}{g_{j}(b)^{+}-g_{j}(b)^{-}}+\left(1-\frac{\left.g_{j}(a)^{+}+q_{j}\right)-g_{j}(b)^{-}}{g_{j}(b)^{+}-g_{j}(b)^{-}}\right)=1
$$

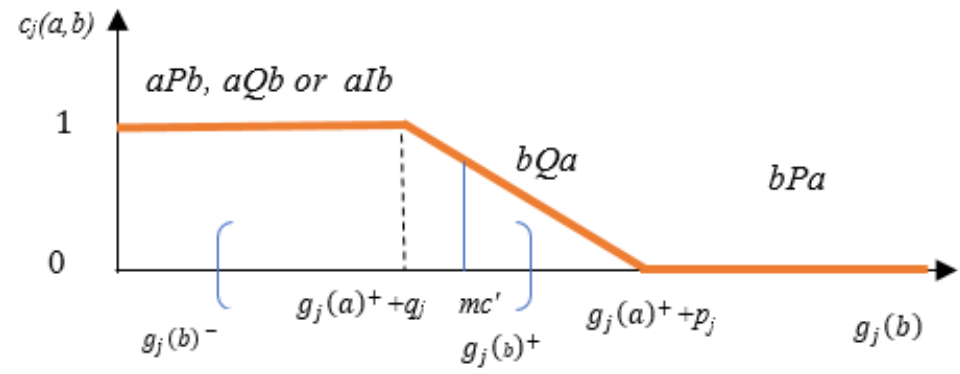

Figure 6. Partial concordance calculation -case 4-

Case 5: In this fifth case, we consider that the UI of alternative $b$ overlaps the weak preference area in favor of $b(b Q a)$ and the strict preference area in favor of alternative $b(b P a)$. In this case, the concordance $c_{j}(a, b)$ must take into account the concordance $c_{j 3}(a, b)$ of the portion of the UI of $b$ that is included in the weak preference area $(b Q a)$, and the concordance $c_{j 4}(a, b)$ of the portion which is included in the strict preference area $(b P a)$. See Figure 7.

More precisely, if $g_{j}(a)^{+}+q_{j}<g_{j}(b)^{-}<g_{j}(a)^{+}+p_{j}$ and $g_{j}(b)^{+}>g_{j}(a)^{+}+p_{j}$ then $c_{j}(a, b)=c_{j 3}(a, b)+$ $c_{j 4}(a, b)$.

Where:

- $c_{j 3}(\mathrm{a}, \mathrm{b})$ expresses the portion of the concordance of the interval $\left[g j(b)^{-}, g_{j}(a)^{+}+p_{j}\right]$ that is included in the weak preference area of $b(b Q a)$. For the calculation of $c_{j 3}(a, b)$, we apply the case 2 . Which takes as concordance an interpolation of the medium of the interval $\left[g_{j}(b)^{-}, g_{j}(a)^{+}+p_{j}\right]$ as shown in Figure 7. However, the concordance $c_{j 3}(a, b)$ must be attenuated by the region of the UI of $b$ outside the area $b Q a$.

$-c_{j 4}(\mathrm{a}, \mathrm{b})$ expresses the portion of the concordance of the interval $\left[g_{j}(a)^{+}+p_{j}, g j(b)^{+}\right]$that is included in the strict preference area of $b(b P a)$. For the calculation of $c j 4(a, b)$, we apply the case 3 , which takes as concordance a value equal to 0 .

So we then take in this fifth case :

$$
c_{j 3}(a, b)=\left(1-\frac{g_{j}(b)^{+}-\left(g_{j}(a)^{+}+p_{j}\right)}{g_{j}(b)^{+}-g_{j}(b)^{-}}\right) \times \frac{g_{j}(a)^{+}+p_{j}-m c^{\prime \prime}}{p_{j}-q_{j}} \text { and } c_{j 4}(a, b)=0
$$

Where $m c^{\prime \prime}=\frac{g_{j}(b)^{-}+g_{j}(a)^{+}+p_{j}}{2}$ is the medium of the interval $\left[g_{j}(b)^{-}, g_{j}(a)^{+}+p_{j}\right]$.

Case 6: In this sixth and last case of partial concordance computation, we suppose that the UI of alternative $b$ overlaps with the three preference areas: the first area in favor of $a(a P, a Q b, a I b)$, the second weakly preference 


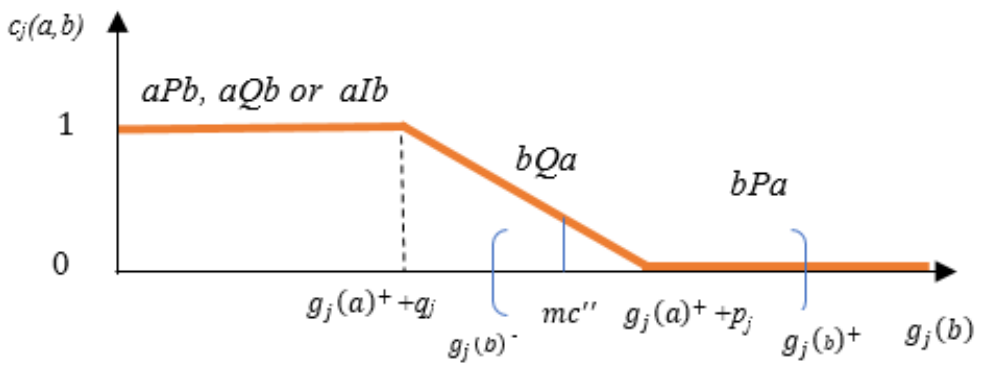

Figure 7. Partial concordance calculation -case 5-

area in favor of $b(b Q a)$, and the third strictly preference area in favor of $b(b P a)$. The concordance $c_{j}(a, b)$ is thus calculated as a function of these three overlaps. We consider $\operatorname{cj} 5(a, b)$ is the concordance of the intersection of the UI with the preference area in favor of the alternative $a . \operatorname{cj} 6(a, b)$ is the concordance of the intersection part of the UI with the weak preference area in favor of the alternative $b$, and $c j 7(a, b)$ is the concordance of the intersection part of the UI with the strict preference area in favor of the alternative $b$. See Figure 8.

More precisely, if $g_{j}(b)^{-}<g_{j}(a)^{+}+q_{j}$ and $g_{j}(b)^{+}>g_{j}(a)^{+}+p_{j}$ then $c_{j}(a, b)=c_{j 5}(a, b)+c_{j 6}(a, b)+c_{j 7}(a, b)$. With:

$-c_{j 5}(a, b)$ expresses the portion of the concordance of the interval $\left[g_{j}(b)^{-}, g_{j}(a)^{+}+q_{j}\right]$ that is included in the area in favor the alternative $a$. For the calculation of $c_{j 5}(a, b)$, we apply precisely the same formula used for $c_{j 1}(a, b)$. $c_{j 6}(a, b)$ expresses the portion of the concordance of the interval $\left[g_{j}(a)^{+}+q_{j}, g_{j}(a)^{+}+p_{j}\right]$ that is included in the weak preference area of $b(b Q a)$. For the calculation of $c_{j 6}(a, b)$, we apply the case 2 . Which takes as concordance an interpolation of the medium of the whole interval $\left[g_{j}(a)^{+}+q_{j}, g_{j}(a)^{+}+p_{j}\right]$. The value of $c_{j 6}(a, b)$ must be attenuated by the portion of the interval outside this area by the value:

$1-\frac{\left(g_{j}(a)^{+}+q_{j}\right)-g_{j}(b)^{-}}{g_{j}(b)^{+}-g_{j}(b)^{-}}+\frac{\left.g_{j}(b)^{+}-\left(g_{j}(a)^{+}\right)+p_{j}\right)}{g_{j}(b)^{+}-g_{j}(b)^{-}}$. Or strengthened by the value: $\frac{\left(g_{j}(a)^{+}+p_{j}\right)-\left(g_{j}(a)^{+}+q_{j}\right)}{g_{j}(b)^{+}-g_{j}(b)^{-}}=$ $\frac{p_{j}-q_{j}}{g_{j}(b)^{+}-g_{j}(b)^{-}}$

Because we have:

$$
\frac{\left(g_{j}(a)^{+}+q_{j}\right)-g_{j}(b)^{-}}{g_{j}(b)^{+}-g_{j}(b)^{-}}+\frac{\left(g_{j}(a)^{+}+p_{j}\right)-\left(g_{j}(a)^{+}+q_{j}\right)}{g_{j}(b)^{+}-g_{j}(b)^{-}}+\frac{g_{j}(b)^{+}-\left(g_{j}(a)^{+}+p_{j}\right)}{g_{j}(b)^{+}-g_{j}(b)^{-}}=1
$$

- $c 7(a, b)$ expresses the portion of the concordance of the interval $\left[g_{j}(a)^{+}+p_{j}, g_{j}(b)^{+}\right]$that is included in the strict preference area of $b(b P a)$. For the calculation of $c j 7(a, b)$, we apply the case 3 , which takes as concordance a value equal to 0 . Then, we take:

$$
\begin{gathered}
c_{j 5}(a, b)=\left(1-\frac{g_{j}(b)^{+}-\left(g_{j}(a)^{+}+q_{j}\right)}{g_{j}(b)^{+}-g_{j}(b)^{-}}\right) \times 1 \\
c_{j 6}(a, b)=\frac{\left(g_{j}(a)^{+}+p_{j}\right)-\left(g_{j}(a)^{+}+q_{j}\right)}{g_{j}(b)^{+}-g_{j}(b)^{-}} \times \frac{\left.g_{j}(a)^{+}+p_{j}-m c^{\prime \prime \prime}\right)}{p_{j}-q_{j}} \\
c_{j 7}(a, b)=0
\end{gathered}
$$

Where $m c^{\prime \prime \prime}=\frac{g_{j}(a)^{+}+q_{j}+g_{j}(a)^{+}+p_{j}}{2}$ is this the medium of the interval $\left[g_{j}(a)^{+}+q_{j}, g_{j}(a)^{+}+p_{j}\right]$.

It can be shown easily that: $\frac{g_{j}(a)^{+}+p_{j}-m c^{\prime \prime \prime}}{p_{j}-q_{j}}=0.5$, whence $c_{j 6}(a, b)=0.5 \times \frac{p_{j}-q_{j}}{g_{j}(b)^{+}-g_{j}(b)^{-}}$. After calculating the partial concordance indices for all the criteria and for all the pairs of alternatives, we calculate the global concordance $c(a, b)$ for all pair of alternatives $a$ and $b$ of the set $A$, as shown in equation (5). 


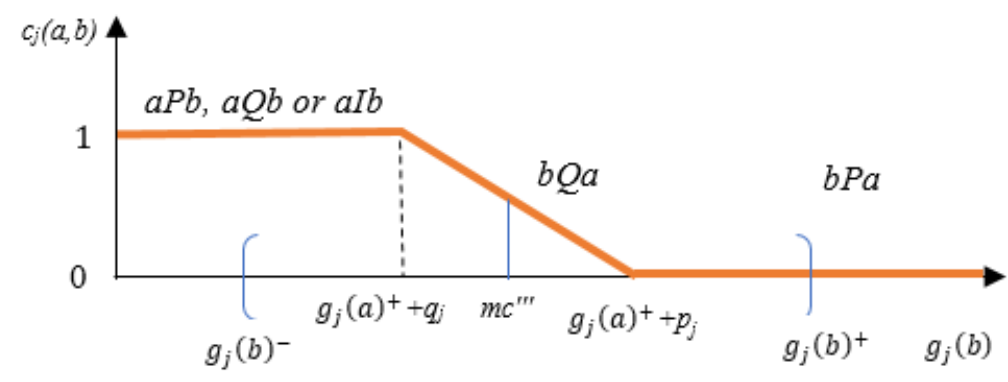

Figure 8. Partial concordance calculation -case 6-

\subsection{Partial discordance calculation}

To calculate the partial discordance index $d_{j}(a, b)$ for each criterion $g_{j}, 1 \leq j \leq m$, and for each pair of alternatives $(a, b)$ of the set $A$, we will use the same approach followed to calculate the partial concordance index $c_{j}(a, b)$, but this time we will follow reverse reasoning. We measure the degree of whether the UI of $b$ is preferred or indifferent to that of $a$. Simultaneously, we allow the possibility to distinguish the case where the UI of $b$ is widely preferred to the UI of $a$. This case will be noted by $(b P 1 a)$. And the case where the UI of $b$ will be considered as very widely preferred to that of $a$. This case will be noted by $(b P 2 a)$. For these two preferences $P 1$ and $P 2$, we define a veto threshold $v_{j}$, beyond which the discordance is equal to 1 (see Figure 9). Likewise, in the case of the concordance index $c_{j}(a, b)$ computation, we distinguish exactly six cases in total to calculate the discordance index $d_{j}(a, b)$.

Case 1: We start with the case where the UI of alternative $b$ is included in the area in favor of $a(a P b, a Q b, a I b)$, included in the weak preference area of $b(b Q a)$, or even included in the area where there is an overlap with these different areas. We take for this first case $d_{j}(a, b)=0$ because for these areas considered, the UI of the alternative $b$ is located before the two veto areas: the weak veto area $(b P 1 a)$ and the strict veto area $(b P 2 a)$, then no discordance can be assumed. See Figure 9.

More precisely: if $g_{j}(b)^{+} \leq g_{j}(a)^{+}+p_{j}$ then $d_{j}(a, b)=0$.

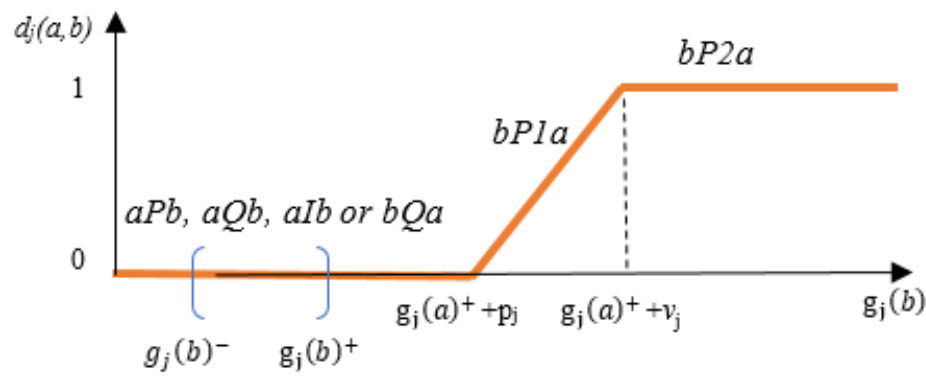

Figure 9. Partial discordance calculation -case 1-

Case 2: In this second case, we examine the point where the UI of $b$ is included in the weak veto area $(b P 1 a)$. We take so a discordance by an increasing linear interpolation, the more that the UI approaches to the strict veto area, the discordance increases towards the maximum discordance, which is equal to 1 . And vice versa, more than the UI of $b$ moves away of the strict veto area, the discordance decreases towards the minimum discordance, which is equal to 0 . The medium $m d$ of the UI of $b$ verifies this property : $m d=\frac{g_{j}(b)^{-}+g_{j}(b)^{+}}{2}$. See Figure 10.

More precisely: If $g_{j}(a)^{+}+p_{j}<g_{j}(b)^{+}<g_{j}(a)^{+}+v_{j}$ and $g_{j}(b)^{-}>g_{j}(a)^{+}+p_{j}$ then, $d_{j}(a, b)=$ $\frac{m d-\left(g_{j}(a)^{+}+p_{j}\right)}{v_{j}-p_{j}}$. 


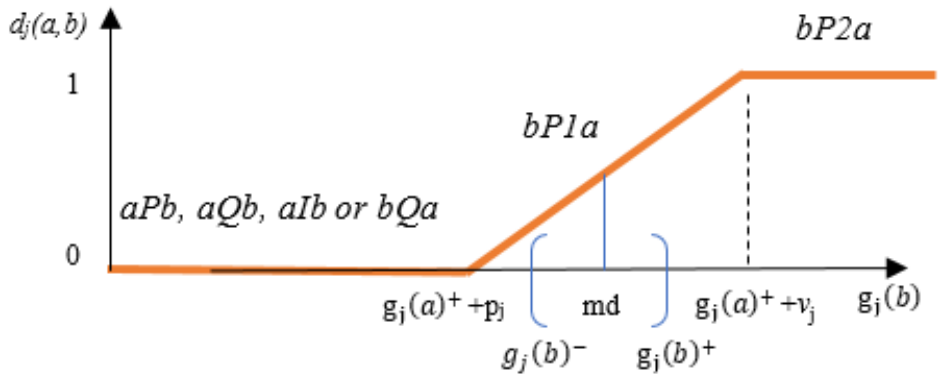

Figure 10. Partial discordance calculation -case 2-

Case 3: In this third case, we trait the case where the UI of alternative $b$ is located beyond the strict veto area $(b P 2 a)$. In this case, it is clear to take the minimal discordance: $d_{j}(a, b)=0$. See Figure 11. More precisely: If $g_{j}(b)^{-}>g_{j}(a)^{+}+v_{j}$ then, $d_{j}(a, b)=1$.

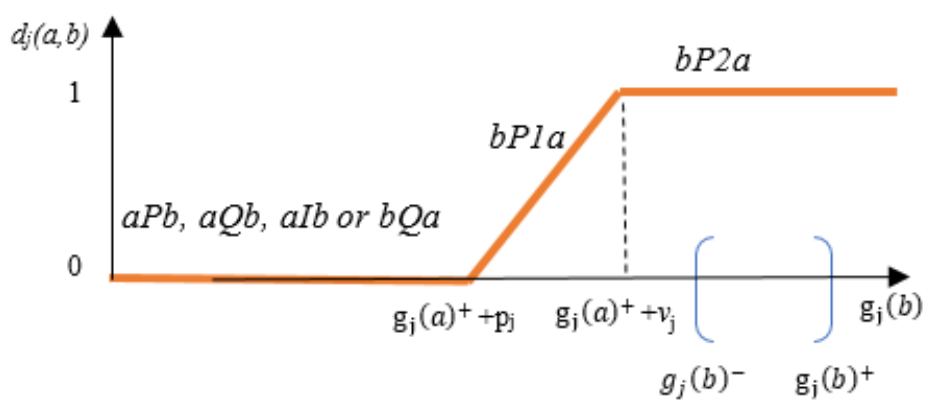

Figure 11. Partial discordance calculation -case 3-

Case 4: In this fourth case, we consider that the UI of alternative $b$ overlaps the area designated by $(a P b, a Q b$ or $a I b)$ and the weak veto area: $b P 1 a$. In this case, the discordance $d_{j}(a, b)$ must take into account the discordance $d_{j 1}(a, b)$ of the portion of the UI of $b$ that is included in the area $(a P b, a Q b$ or $a I b)$, and the discordance $d_{j 2}(a, b)$ of the portion that is included in area $(b P 1 a)$. See Figure 12.

More precisely: if $g_{j}(b)^{-} \leq g_{j}(a)^{+}+p_{j} \quad$ and $\quad g_{j}(a)^{+}+p_{j}<g_{j}(b)^{+}<g_{j}(a)^{+}+v_{j} \quad$ then $\quad d_{j}(a, b)=$ $d_{j 1}(a, b)+d_{j 2}(a, b)$.

With:

$-d_{j 1}(a, b)$ expresses the portion of the discordance of the interval $\left[g_{j}(b)^{-}, g j(a)^{+}+p_{j}\right]$, which is included in the area in favor of $a$ and the weak preference area of $b$. For the calculation of $d_{j 1}(a, b)$, we apply the case 1 , which takes a discordance $d_{j 1}(a, b)=0 .-d j 2(a, b)$ expresses the portion of the discordance of the interval $\left[g_{j}(a)^{+}+p_{j}, g_{j}(b)^{+}\right]$that is included in the weak veto area $(b P 1 a)$. For the calculation of $d_{j 2}(a, b)$, we apply case 2. Which takes as discordance an interpolation of the medium of the interval $\left[g_{j}(a)^{+}+p_{j}, g_{j}(b)^{+}\right]$. But as in the case of the concordance, the value of $d_{j 2}=(a, b)$ should be attenuated from the portion of the interval that is outside of this area by the value: $1-\frac{\left(g_{j}(a)^{+}+p_{j}\right)-g_{j}(b)^{-}}{g_{j}(b)^{+}-g_{j}(b)^{-}}$. Or reinforced by the value $: \frac{g_{j}(b)^{+}-\left(g_{j}(a)^{+}+p_{j}\right)}{g_{j}(b)^{+}-g_{j}(b)^{-}}$. Because, $\frac{\left(g_{j}(a)^{+}+p_{j}\right)-g_{j}(b)^{-}}{g_{j}(b)^{+}-g_{j}(b)^{-}}+\frac{g_{j}(b)^{+}-\left(g_{j}(a)^{+}+p_{j}\right)}{g_{j}(b)^{+}-g_{j}(b)^{-}}=1$. We take so:

$$
d_{j 1}(a, b)=0
$$




$$
d_{j 2}(a, b)=\left(1-\frac{\left(g_{j}(a)^{+}+p_{j}\right)-g_{j}(b)^{-}}{g_{j}(b)^{+}-g_{j}(b)^{-}}\right) \times \frac{m d^{\prime}-\left(g_{j}(a)^{+}+p_{j}\right)}{v_{j}-p_{j}}
$$

Where $m d^{\prime}$ is the medium of the interval $\left[g_{j}(a)^{+}+p_{j}, g_{j}(b)^{+}\right]: m d^{\prime}=\frac{g_{j}(a)^{+}+p_{j}+g_{j}(b)^{+}}{2}$.

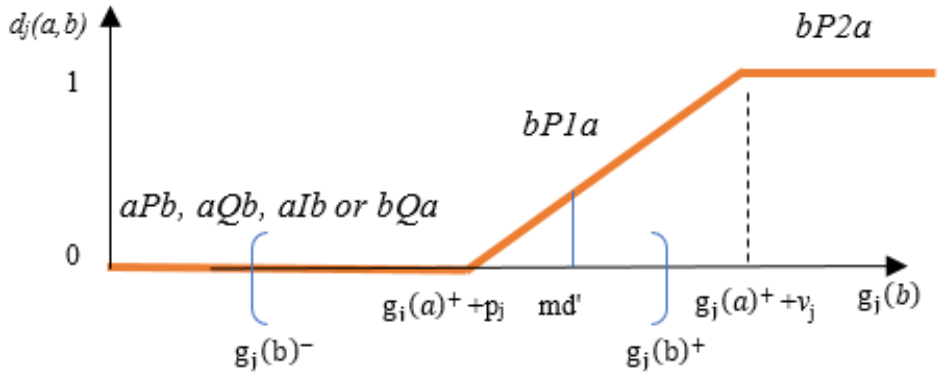

Figure 12. Partial discordance calculation -case 4-

Case 5: In this fifth case, we consider that the UI of alternative $b$ overlaps the weak veto area: $b P 1 a$ and the strict veto area: $b P 2 a$. In this case, the discordance $d_{j}(a, b)$ must take into account the discordance $d_{j 3}(a, b)$ of the portion of the UI of $b$ that is included in the weak veto area, and the discordance $d_{j 4}(a, b)$ of the portion which is included in the strict veto area. See Figure 13.

More precisely: if $g_{j}(a)^{+}+p_{j}<g_{j}(b)^{-}<g_{j}(a)^{+}+v_{j}$ and $g_{j}(b)^{+}>g_{j}(a)^{+}+v_{j}$ then $d_{j}(a, b)=d_{j 3}(a, b)+$ $d_{j 4}(a, b)$.

With:

$-d_{j 3}(a, b)$ expresses the portion of the discordance of the interval $\left[g_{j}(b)^{-}, g_{j}(a)^{+}+v_{j}\right]$ that is included in the weak veto area $(b P 1 a)$. For the calculation of $d_{j 3}(a, b)$, we apply the case 2 , which takes as discordance an increasing interpolation of the medium of the interval $\left[g_{j}(b)^{-}, g_{j}(a)^{+}+v_{j}\right]$, but this value must be attenuated by the portion of the interval outside this area by the value: $1-\frac{g_{j}(b)^{+}-\left(g_{j}(a)^{+}+v_{j}\right)}{g_{j}(b)^{+}-g_{j}(b)^{-}}$. Or reinforced by the value $: \frac{\left(g_{j}(a)^{+}+v_{j}\right)-g_{j}(b)^{-}}{g_{j}(b)^{+}-g_{j}(b)^{-}}$. - $d_{j 4}(a, b)$ expresses the portion of the concordance of the interval $\left[g_{j}(a)^{+}+v_{j}, g_{j}(b)^{+}\right]$that is included in the strict veto area $(b P 2 a)$. For the calculation of $d_{j 4}(a, b)$, we apply the case 3 , which takes as a discordance equal to 1 . This value must be attenuated by the portion of the interval which is outside this area by the value: $1-\frac{\left(g_{j}(a)^{+} v_{j}\right)-g_{j}(b)^{-}}{g_{j}(b)^{+}-g_{j}(b)^{-}}$. Or reinforced by the value : $\frac{g_{j}(b)^{+}-\left(g_{j}(a)^{+}+v_{j}\right)}{g_{j}(b)^{+}-g_{j}(b)^{-}}$.

We take so :

$$
\begin{gathered}
d_{j 3}(a, b)=\left(1-\frac{g_{j}(b)^{+}-\left(g_{j}(a)^{+}+v_{j}\right)}{g_{j}(b)^{+}-g_{j}(b)^{-}}\right) \times \frac{m d^{\prime \prime}-\left(g(a)^{+}+p_{j}\right)}{v_{j}-p_{j}} \\
d_{j 4}(a, b)=\left(1-\frac{\left(g_{j}(a)^{+}+v_{j}\right)-g_{j}(b)^{-}}{g_{j}(b)^{+}-g_{j}(b)^{-}}\right) \times 1
\end{gathered}
$$

Where: $m d^{\prime \prime}$ is the medium of the interval $\left[g_{j}(b)^{-}, g_{j}(a)^{+}+v_{j}\right]: m d^{\prime \prime}=\frac{\left(g_{j}(b)^{-}+g_{j}(a)^{+}+v_{j}\right.}{2}$.

Case 6: In this sixth and the last case of partial discordance computation, we consider that the UI of alternative $b$ overlaps the three areas: the area in favor of $a$ or weakly in favor of $b:(a P b, a Q b, a I b o r b Q a)$, the weak veto area: $b P 1 a$, and the strict veto area: $b P 2 a$. In this case, the discordance $d_{j}(a, b)$ must take into account three discordance: the discordance $d_{j 5}(a, b)$ of the portion of the UI of $b$ which is included in the area "aPb,aQb,aIborbQa", the discordance $d_{j 6}(a, b)$ of the portion of the UI of $b$ that is included in the weak veto area, and the discordance $d_{j 7}(a, b)$ of the portion of the IU of $b$ that is included in the strict veto area. See Figure 14.

More precisely, if $g_{j}(b)^{-} \leq g_{j}(a)^{+}+p_{j}$ and $g_{j}(b)^{+}>g_{j}(a)^{+}+v_{j}$ then $d_{j}(a, b)=d_{j 5}(a, b)+d_{j 6}(a, b)+$ $d_{j 7}(a, b)$ 


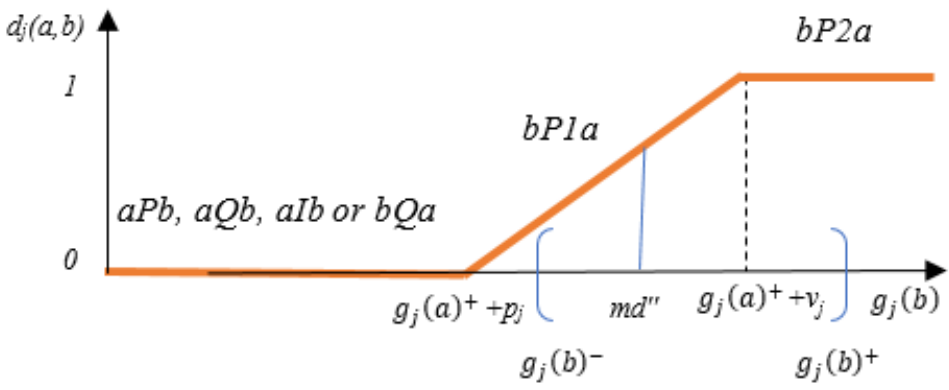

Figure 13. Partial discordance calculation -case 5-

With:

- $d_{j 5}(a, b)$ corresponds to the discordance of the interval $\left[g_{j}(b)^{-}, g_{j}(a)^{+}+p_{j}\right]$ which is included in the area in favor of $a$ or weakly in favor of $b$ : " $a P b, a Q b, a I b$ or $b Q a$ ". In this case, we take so:

$$
d_{5}(a, b)=0
$$

- $d_{j 6}(a, b)$ expresses the portion of the discordance of the interval $\left[g_{j}(a)^{+}+p_{j}, g_{j}(a)^{+}+v_{j}\right]$ which is equal to the whole weak veto area: $b P 1 a$. As for calculating the value of concordance $c j 6(a, b)$, we obtain so:

$$
d_{j 6}(a, b)=0.5 \times \frac{v_{j}-p_{j}}{\left(g_{j}(b)^{+}-g_{j}(b)^{-}\right)}
$$

- $d_{j 7}(a, b)$ expresses the portion of the discordance of the interval $\left[g_{j}(a)^{+}+v_{j}, g_{j}(b)^{+}\right]$that is included in the strict veto area: $b P 2 a$. As for the calculation of the discordance $d_{j 4}(a, b)$, we obtain :

$$
d_{j 7}(a, b)=\frac{g_{j}(b)^{+}-\left(g_{j}(a)^{+}+v_{j}\right)}{g_{j}(b)^{+}-g_{j}(b)^{-}}
$$

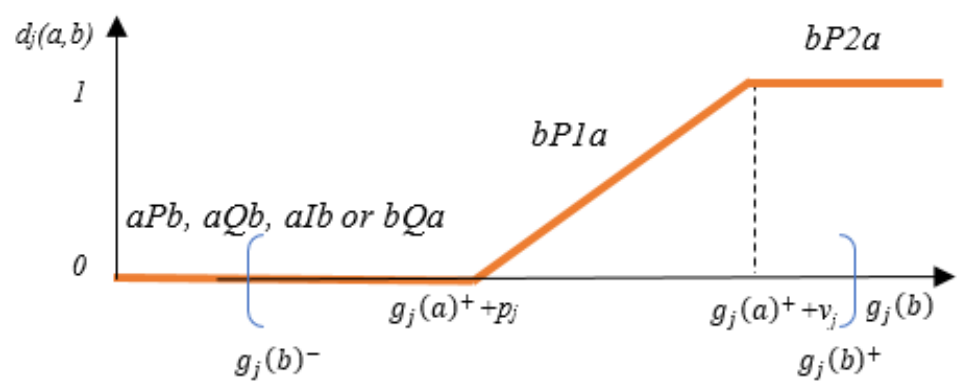

Figure 14. Partial discordance calculation -case 6-

\section{Experimentation of the ELECTRE III method extension}

\subsection{Presentation of the experimentation example}

Roger and all. [28] have shown the ELECTRE method's ability to solve MCDM problems of economic, technical, and environmental dimensions. They gave a case study that illustrates the different aspects of the decision in the 
ecological project planning process: definition of objectives, identification of alternatives, a description of criteria, evaluation of alternatives, and the final recommendation. To illustrate the proposed approach, we give an example inspired by this real planning of the environmental project in this paragraph. In this example, we consider precisely three projects $(A 1, A 2, A 3)$ competing to install an oil refinery. The problem is to choose the best project to retain the oil refinery facility. For this, each project is then evaluated based on four environmental criteria: Cr1: Noise pollution Cr2: Separation of territory Cr3: Air Pollution Cr4: Impact on land use planning In this decision problem and given the nature of the criteria considered, it is difficult for the DM to clearly and precisely express each project's evaluations. This makes the use of the ELECTRE III method impossible. To solve this problem, we propose applying the revised version of the ELECTRE III method suitable for the cases of preferences expressed using the UIs.

\subsection{Application of the proposed approach}

To show the efficiency and the feasibility of the ELECTRE III method extension thus proposed, we apply this approach to rank the three oil station installation projects from the best to the worst project.

Step 1: Elaboration of the decision matrix: Table 2 presents the decision matrix, where each performance $g_{j}(a)$ is expressed by anUI $\left[g_{j}(a)^{-}, g_{j}(a)^{+}\right]$. The matrix is given in the following table 2. As shown in Table 2, all

Table 2. Performances matrix

\begin{tabular}{|c|c|c|c|c|}
\hline Alternatives-Criteria & Cr1:g1 & Cr2:g2 & Cr3:g3 & Cr4:g4 \\
\hline A1 & {$[6,12]$} & {$[18,25]$} & {$[4,6]$} & {$[9,11]$} \\
\hline A2 & {$[7,9]$} & {$[27,28]$} & {$[4,6]$} & {$[8,12]$} \\
\hline A3 & {$[6,8]$} & {$[9,12]$} & {$[2,4]$} & {$[6,8]$} \\
\hline
\end{tabular}

the preferences of the three alternatives $A 1, A 2, A 3$ are uncertain and expressed by UIs. For criterion $\mathrm{Cr} 1$, noise pollution, the DM expresses his preferences in the form of intervals. For example, for the alternative $A 1$, the DM provides an interval of $[6,12]$. Depending on this, this alternative obtains a noise judgment that varies between the values 6 and 12 .

- Step 2: Estimation of the parameters For this illustration example, we choose fixed thresholds, see Table 3. For a suitable choice of these thresholds, we will estimate them according to the extent of each criterion, to do this, we use the following calculation formulas:

- Choice of the preference threshold $q_{j}=10 \%$ of the difference on the criterion:

$q_{j}=\left(\max _{a \in A}\left(g_{j}(a)^{+}\right)-\min _{a \in A}\left(g_{j}(a)^{-}\right) \times 0.1\right.$ - Choice of the preference threshold $p_{j}=30 \%$ of the difference on the criterion:

$p_{j}=\left(\max _{a \in A}\left(g_{j}(a)^{+}\right)-\min _{a \in A}\left(g_{j}(a)^{-}\right) \times 0.3\right.$ - Choice of the preference threshold $v_{j}=70 \%$ of the difference on the criterion:

$v_{j}=\left(\max _{a \in A}\left(g_{j}(a)^{+}\right)-\min _{a \in A}\left(g_{j}(a)^{-}\right) \times 0.7\right.$

These percentages are given and may be modified for the purpose of the robustness analysis (Table 4 and 5).

Table 3. Thresholds and weights of criteria

\begin{tabular}{|c|c|c|c|c|}
\hline Criteria & g1 & g2 & g3 & g4 \\
\hline Weights $w_{j}$ & 3 & 2 & 4 & 1 \\
\hline Indifference thresholds $\left(q_{j}\right)$ & 0.6 & 1.9 & 0.4 & 0.5 \\
\hline preference thresholds $\left(p_{j}\right)$ & 1.8 & 5.7 & 1.2 & 2.1 \\
\hline Veto thresholds $\left(v_{j}\right)$ & 4.2 & 13.3 & 2.8 & 3.85 \\
\hline
\end{tabular}


Table 4. Area of indifference

\begin{tabular}{|c|c|c|c|c|}
\hline Alternatives-Criteria & $g_{1}(a)^{-}-q_{1}$ & $g_{1}(a)^{+}+q_{1}$ & $g_{2}(a)^{-}-q_{2}$ & $g_{2}(a)^{+}+q_{2}$ \\
\hline A1 & 5.4 & 12.6 & 16.1 & 26.9 \\
\hline A2 & 6.4 & 9.6 & 25.1 & 29.9 \\
\hline A3 & 5.4 & 8.6 & 7.1 & 13.9 \\
\hline \hline Alternatives-Criteria & $g_{3}(a)^{-}-q_{3}$ & $g_{3}(a)^{+}+q_{3}$ & $g_{4}(a)^{-}-q_{4}$ & $g_{4}(a)^{+}+q_{4}$ \\
\hline A1 & 3.6 & 6.4 & 8.5 & 11.5 \\
\hline A2 & 3.6 & 6.4 & 7.5 & 12.5 \\
\hline A3 & 1.6 & 4.4 & 5.5 & 8.5 \\
\hline
\end{tabular}

Table 5. Area of preferences and veto

\begin{tabular}{|c|c|c|c|c|}
\hline Alternatives-Criteria & $g_{1}(a)^{-}+p_{1}$ & $g_{1}(a)^{+}+v_{1}$ & $2(a)^{-}+p_{2}$ & $g_{2}(a)^{+}+v_{2}$ \\
\hline A1 & 13.8 & 16.2 & 3.07 & 38.3 \\
\hline A2 & 10.8 & 13.2 & 33.7 & 41.3 \\
\hline A3 & 9.8 & 12.2 & 17.7 & 25.3 \\
\hline \hline Alternatives-Criteria & $g_{3}(a)^{-}+p_{3}$ & $g_{3}(a)^{+}+v_{3}$ & $g_{4}(a)^{-}+p_{4}$ & $g_{2}(a)^{+}+v_{4}$ \\
\hline A1 & 7.2 & 8.8 & 13.1 & 14.85 \\
\hline A2 & 7.2 & 8.8 & 14.1 & 15.85 \\
\hline A3 & 5.2 & 6.8 & 10.1 & 11.85 \\
\hline
\end{tabular}

Step 3: Determination of partial concordance indices We present the concordance indices for each criterion in Table 6, Table 7, Table 8, and Table 9.

Step 4: Determination of partial discordance indices The discordance indices for each criterion are obtained and

Table 6. The concordance indices of criterion 1

\begin{tabular}{|c|c|c|c|}
\hline Criterion 1 & A1 & A2 & A3 \\
\hline A1 & 0 & 1 & 1 \\
A2 & 0.8 & 0 & 1 \\
A3 & 0.533 & 0.966 & 0 \\
\hline \hline
\end{tabular}

Table 7. The concordance indices of criterion 2

\begin{tabular}{|c|c|c|c|}
\hline Criterion 2 & A1 & A2 & A3 \\
\hline A1 & 0 & 0.8421 & 1 \\
A2 & 1 & 0 & 1 \\
A3 & 0 & 0 & 0 \\
\hline \hline
\end{tabular}

presented in Table 10, Table 11, Table 12, Table 13. 
Table 8. The concordance indices of criterion 3

\begin{tabular}{|c|c|c|c|}
\hline Criterion 3 & A1 & A2 & A3 \\
\hline A1 & 0 & 1 & 1 \\
A2 & 1 & 0 & 1 \\
A3 & 0.4 & 0.4 & 0 \\
\hline \hline
\end{tabular}

Table 9. The concordance indices of criterion 4

\begin{tabular}{|c|c|c|c|}
\hline Criterion 4 & A1 & A2 & A3 \\
\hline A1 & 0 & 0.98 & 1 \\
A2 & 1 & 0 & 1 \\
A3 & 0.189 & 0.325 & 0 \\
\hline \hline
\end{tabular}

Table 10. The discordance matrix of criterion 1

\begin{tabular}{|c|c|c|c|}
\hline Criterion 1 & $\mathrm{A} 1$ & $\mathrm{~A} 2$ & $\mathrm{~A} 3$ \\
\hline $\mathrm{A} 1$ & 0 & 0 & 0 \\
$\mathrm{~A} 2$ & 0.15 & 0 & 0 \\
$\mathrm{~A} 3$ & 0.168 & 0 & 0 \\
\hline \hline
\end{tabular}

Table 11. The discordance matrix of criterion 2

\begin{tabular}{|c|c|c|c|}
\hline Criterion 2 & A1 & A2 & A3 \\
\hline A1 & 0 & 0 & 0 \\
A2 & 0 & 0 & 0 \\
A3 & 0.5 & 1 & 0 \\
\hline \hline
\end{tabular}

Table 12. The discordance matrix of criterion 3

\begin{tabular}{|c|c|c|c|}
\hline Criterion 3 & A1 & A2 & A3 \\
\hline A1 & 0 & 0 & 0 \\
A2 & 0 & 0 & 0 \\
A3 & 0.1 & 0.1 & 0 \\
\hline \hline
\end{tabular}

Table 13. The discordance matrix of criterion 4

\begin{tabular}{|c|c|c|c|}
\hline Criterion 4 & A1 & A2 & A3 \\
\hline A1 & 0 & 0 & 0 \\
A2 & 0 & 0 & 0 \\
A3 & 0.12 & 0.257 & 0 \\
\hline
\end{tabular}

Stat., Optim. Inf. Comput. Vol. 10, February 2022 
Step 5: Calculation of the global concordance matrix The matrix of global concordance is calculated by (5) and given in Table 14.

Table 14. The concordance matrix

\begin{tabular}{|c|c|c|c|}
\hline Alternatives & A1 & A2 & A3 \\
\hline A1 & 0 & 0.96632 & 1 \\
A2 & 0.94 & 0 & 1 \\
A3 & 0.3388 & 0.4823 & 0 \\
\hline
\end{tabular}

Step 6: Calculation of degrees of credibility: The matrix of degrees of credibility, given in Table 15, is calculated by equation 7 .

Note that for each pair $(a, b)$ of alternatives and for each criterion $g_{j}$, all the indices of discordance $d_{j}(a, b)$ are lower than the index of concordance $c(a, b)$, which gives degrees of credibility coinciding with the concordance indices: $\rho(a, b)=c(a, b)$.

Table 15. Matrix of credibility degrees

\begin{tabular}{|c|c|c|c|}
\hline Alternatives & A1 & A2 & A3 \\
\hline A1 & 0 & 0.96632 & 1 \\
A2 & 0.94 & 0 & 1 \\
A3 & 0.3388 & 0.4823 & 0 \\
\hline
\end{tabular}

Step 7: Establishment of descending and ascending pre-order We deduce the descending and ascending pre-order from the matrix of credibility degrees and the calculation of the qualification scores (Figure 15).

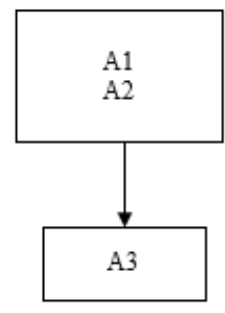

(a)

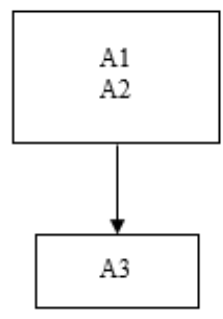

(b)

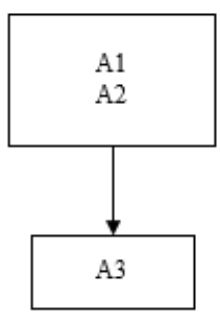

(c)

Figure 15. (a) ascending distillation, (b) descending distillation, (c) outranking graph $S$.

Step 8: Establishment of a median pre-order, giving the final ranking The descending and ascending pre-orders are combined into a median pre-order to build the desired pre-order (Table 16) finally.

\subsection{Discussion}

To rank all three projects $A=\{A 1, A 2, A 3\}$, four criteria are then used $F=\{g 1, g 2, g 3, g 4\}$. All these criteria are chosen to select the oil station that will preserve and compensate as much as possible for environmental and territorial development. Table 16 gives the result of the final ranking calculated by the proposed approach. In these illustrative calculations, where we have considered all the criteria to be maximized, we obtain A1 and A2 as best projects tied, while alternative A3 is ranked last as the worst project. Note that the criteria considered in this 
Table 16. The final ranking

\begin{tabular}{|c|c|}
\hline \hline Alternatives & Ranks \\
\hline A1 & 1 \\
A2 & 1 \\
A3 & 2 \\
\hline
\end{tabular}

example are really must be minimized. We will obtain that alternative A3 will ultimately be the best oil refinery installation project with similar calculations for the maximization case.

\section{Conclusions}

Numerous decision-making problems require taking into account the conflicting views, uncertainties, and imprecise judgment of decision-makers. This paper has proposed a new approach inspired by the same calculation process used in the ELECTRE III method, but it supports uncertain and imprecise preferences. The extension offers decision-makers to express their uncertain judgments by uncertainty intervals, as in experimental measurements in physical or chemical sciences. Besides, as for the ELECTRE III method, the proposed method can take into account the hesitation between indifference and preference in the comparisons of the intervals of uncertainties. The illustrative example shows that all the formulas for calculating the two concordance indices and the discordance are well representative and relevant, thus covering all possible uncertainty intervals. In the extension of the ELECTRE III method proposed, we only consider the case where uncertainty intervals express all the evaluations. However, for a general case, we may have a multi-criteria decision problem where the decision matrix $\mathrm{M}$ evaluations are mixed between exact preferences and uncertain preferences. In this case, all the calculation rules proposed for the uncertain case can be generalized. Indeed, for the case of the comparison of two values expressed by intervals of uncertainty, the approach presented is then applied. For the case of the comparison of two exact evaluations, we use the basic method ELECTRE III. And finally, for the possibility of comparing an interval with an exact value, for example, the exact value g(a) will be replaced by the interval $\left[g(a)^{-}, g(a)^{+}\right]$with $g(a)^{-}=g(a)^{+}$, then apply the same comparison rules suggested in the extension. Lastly, our future work will focus on applying the promising results obtained in this present paper and adapting the uncertain preferences to other MCDM methods such as the TOPSIS [29] and PROMETHEE methods.

\section{REFERENCES}

1. Bernard Roy, Denis Bouyssou, Aide multicritère méthodes et cas, Economica, Paris, 1993.

2. L.A. Zadeh, "Fuzzy sets," Information and Control, 8(3), 338-353, 1965, doi:10.1016/S0019-9958(65)90241-X.

3. Lucien Yves Maystre, Jacques Pictet, Jean Simos, Méthodes multicritères ELECTRE (Description, conseils pratiques et cas d'application à la gestion environnementale), Eyrolles, 1994.

4. B. Roy, ELECTRE III Algorithme de Classement Basé sur une Représentation Floue des Préférences en Présence de Critères Multiples," Cahiers du CERO, Vol. 20, No. 1, 1978, pp. 3-24.

5. J. Figueira, V. Mousseau, B. Roy, ELECTRE methods, Springer New York LLC: 133-162, 2005, doi:10.1007/0-387-23081-5-4.

6. F.Z. El Mazouri, M.C. Abounaima, K. Zenkouar, A.E.H. Alaoui, "Application of the ELECTRE III Method at the Moroccan Rural Electrification Program," International Journal of Electrical and Computer Engineering (IJECE), 8(5), 3285-3295, 2018, doi:10.11591/ijece.v8i5.pp3285-3295.

7. M. Rogers, M. Bruen, "Choosing realistic values of indifference, preference and veto thresholds for use with environmental criteria within ELECTRE," European Journal of Operational Research, 107(3), 542-551, 1998, doi:10.1016/S0377-2217(97)00175-6.

8. A. Hatami-Marbini, M. Tavana, M. Moradi, F. Kangi, "A fuzzy group Electre method for safety and health assessment in hazardous waste recycling facilities," Safety Science, 51(1), 414-426, 2013, doi:10.1016/j.ssci.2012.08.015.

9. J. Ramos, L. Bearzotti, E. Milani, F. Woscoff, M. Gorsky, C.M. Carletti, M.R. Galli, O. Chiotti, "Decision support systems generator for industrial companies Module I: Product design support system," Computers and Industrial Engineering, 35(1-2), 303-306, 1998, doi:10.1016/S0360-8352(98)00080-1. 
10. A. Mendas, A. Delali, "Integration of MultiCriteria Decision Analysis in GIS to develop land suitability for agriculture: Application to durum wheat cultivation in the region of Mleta in Algeria," Computers and Electronics in Agriculture, 83, 117-126, 2012, doi:10.1016/j.compag.2012.02.003.

11. G.D. Betrie, R. Sadiq, K.A. Morin, S. Tesfamariam, "Selection of remedial alternatives for mine sites: A multicriteria decision analysis approach," Journal of Environmental Management, 119, 36-46, 2013, doi:10.1016/j.jenvman.2013.01.024.

12. M. Mousavi, H. Gitinavard, S.M. Mousavi, A soft computing based-modified ELECTRE model for renewable energy policy selection with unknown information, Renewable and Sustainable Energy Reviews, 68, 774-787, 2017, doi:10.1016/j.rser.2016.09.125.

13. J. Hokkanen, P. Salminen, E. Rossi, M. Ettala, "The choice of a solid waste management system using the Electre II decision-aid method," Waste Management and Research, 13(2), 175-193, 1995, doi:10.1016/S0734-242X(95)90118-3.

14. A. Shahnazari, M. Rafiee, A. Rohani, B. Bhushan Nagar, M.A. Ebrahiminik, M.H. Aghkhani, "Identification of effective factors to select energy recovery technologies from municipal solid waste using multi-criteria decision making (MCDM): A review of thermochemical technologies," Sustainable Energy Technologies and Assessments, 40, 100737, 2020, doi:10.1016/j.seta.2020.100737.

15. M. Ozcalici, M. Bumin, "An integrated multi-criteria decision making model with Self-Organizing Maps for the assessment of the performance of publicly traded banks in Borsa Istanbul," Applied Soft Computing Journal, 90, 106166, 2020, doi:10.1016/j.asoc.2020.106166.

16. E. Karasakal and all, "A multicriteria sorting approach based on data envelopment analysis for project selection problem," Elsevier, Feb. 2021.

17. K. De Brucker, A. Verbeke, C. Macharis, THE APPLICABILITY OF MULTICRITERIA-ANALYSIS TO THE EVALUATION OF INTELLIGENT TRANSPORT SYSTEMS (ITS), Research in Transportation Economics, 8, 151-179, 2004, doi:10.1016/S07398859(04)08008-4.

18. S.S. Hashemi, S.H.R. Hajiagha, E.K. Zavadskas, H.A. Mahdiraji, "Multicriteria group decision making with ELECTRE III method based on interval-valued intuitionistic fuzzy information," Applied Mathematical Modelling, 40(2), 1554-1564, 2016, doi:10.1016/j.apm.2015.08.011.

19. M.C. Wu, T.Y. Chen, "The ELECTRE multicriteria analysis approach based on Atanassov's intuitionistic fuzzy sets," Expert Systems with Applications, 38(10), 12318-12327, 2011, doi:10.1016/j.eswa.2011.04.010.

20. R. Pérez-Fernández, P. Alonso, H. Bustince, I. Díaz, S. Montes, "Applications of finite interval-valued hesitant fuzzy preference relations in group decision making," Information Sciences, 326, 89-101, 2016, doi:10.1016/j.ins.2015.07.039.

21. N. Chen, Z. Xu, "Hesitant fuzzy ELECTRE II approach: A new way to handle multi-criteria decision making problems," Information Sciences, 292, 175-197, 2015, doi:10.1016/j.ins.2014.08.054.

22. F. Roberts, Measurement theory with applications to decision mbing, Utility and the Social Sciences, Addison-We, New York, 1979.

23. P. Vincke, "Preferences and numakers," in: A. Colorni, M. Paruccini, and B. R., ed., A-MCD-A - Aide Multi Critère à la Décision Multiple Criteria Decision Aiding, Joint Research Center: 343-354, 2001.

24. P. Vincke, "P,Q,I-preference structures, Lecture Notes in Economics and Mathematical Systems," In Kacprzyk, J. and Roubens, M. (Eds), Nonconventional Preference Relations in Decision Making, 301, 72-81, 1988.

25. H.F. Li, J.J. Wang, "An improved ranking method for ELECTRE III," in 2007 International Conference on Wireless Communications, Networking and Mobile Computing, WiCOM 2007, IEEE Computer Society: 6659-6662, 2007, doi:10.1109/WICOM.2007.1634.

26. I. Alolyan, "A NEW METHOD FOR COMPARING CLOSED INTERVALS," The Australian Journal of Mathematical Analysis and Applications, 8(1), 1-6, 2011.

27. P. Sevastjanov, P.R.-T. Quarterly, “A probabilistic approach to fuzzy and crisp interval ordering,” Task Quarterly, Vol. 7, No. 1, Pp. $147-156,2003$

28. Martin Gerard Rogers, Michael Bruen, Lucien-Yves Maystre, "Electre and Decision Support: Methods and Applications in Engineering", Google Books, Feb. 2021

29. M.C. Abounaima, L. Lamrini, N. EL Makhfi, M. Ouzarf, "Comparison by Correlation Metric the TOPSIS and ELECTRE II MultiCriteria Decision Aid Methods : Application to the Environmental Preservation in the European Union Countries", Advances in Science, Technology and Engineering Systems Journal, 5(5), 1064-1074, 2020, doi:10.25046/aj0505131. 